\title{
Novel metallic states at low temperatures
}

\author{
S.E. Rowley, R.P. Smith, N. Marcano, M.P.M. Dean, A. Kusmartseva, L.J. Spalek, \\ E.C.T. O'Farrell, D.A. Tompsett, M.L. Sutherland, P.L. Alireza, C. Ko, C. Liu, E. Pugh, \\ S.S. Saxena, and G.G. Lonzarich
}

\author{
Shoenberg Laboratory for Quantum Matter, Cavendish Laboratory, University of Cambridge, \\ J.J. Thomson Ave., Cambridge, CB3 OHE, UK \\ E-mail: ser41@cam.ac.uk
}

Received November 9, 2010

\begin{abstract}
We present an overview of unconventional metallic states arising close to magnetic quantum critical points with a focus on $d$-electron systems. The applicability and potential breakdowns of traditional self-consistent field theories of such materials are discussed as well as related phenomena in other systems.

PACS: 71.10.Ay Fermi-liquid theory and other phenomenological models;

74.20.- $\mathbf{Z}$ Theories and models of superconducting state;

74.25.-q Properties of type I and type II superconductors.
\end{abstract}

Keywords: unconventional metallic states, Fermi-liquid state, large magnetic field, dHvA effect.

\section{Introduction}

The Landau Theory of a Fermi liquid (FL), based on the existence of well-defined low-lying fermionic quasiparticle excitations, has shown remarkable resilience in describing the low temperature properties of a wide range of electrically conducting materials. These include $d$ - and $f$-electron metals, two-dimensional systems, organics, and materials subject to the rather harsh conditions of pressure, magnetic field, disorder and other environmental factors. The robustness of the Fermi-liquid is believed to arise from a kind of topological protection of the low temperature quantum system leading to a sharp Fermi surface in momentum space, even though the interactions between the starting "bare" electrons can be very strong and comparable to their kinetic energies. One of the most effective ways of probing a Fermi-liquid state is to image the Fermi-surface using the de Haas van Alphen (dHvA) effect, a technique pioneered by David Shoenberg at the Cavendish Laboratory [1]. The dHvA measurement is a low frequency probe that can catch, with high precision, even the slowest and heaviest quasiparticles on the verge of localization. Some heavy fermions have masses two or three orders of magnitude greater than the bare electron mass. The figure below (Fig. 1) shows one of the sheets of the Fermi-surface of $\mathrm{YbAlB}_{4}$, measured using dHvA at the Cavendish, where the emergent quasiparticles are heavy at low temperatures. This material is interesting since it provides a particularly striking example of non-Fermi liquid metallic behaviour at low temperatures, including unconventional superconductivity below $80 \mathrm{mK}$ [2].
The dHvA measurement involves placing a metallic sample in a large magnetic field, forcing the electrons into quantized Landau orbits. In the early days of the development of the technique, David Shoenberg was interested in possible corrections to the basic theory. One that was of concern to him involved the role of induced charge currents when a metal is placed in a magnetic field. These can

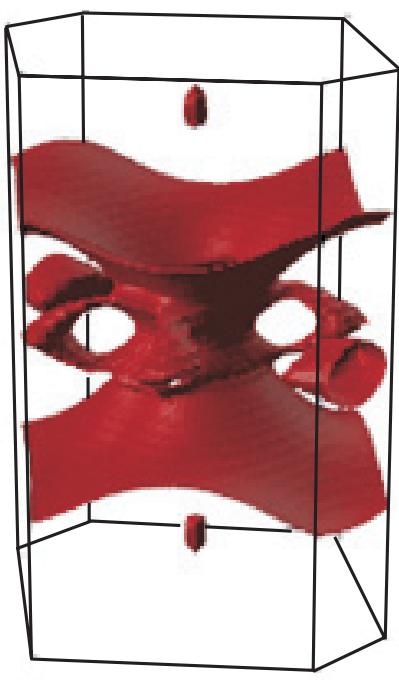

Fig. 1. Fermi-surface of one of the sheets of the heavy fermion compound $\mathrm{YbAlB}_{4}$ determined using the de Haas-van Alphen effect [3]. This is an intermediate valence $f$-electron system, in many ways analogous to $d$-electron metals. Without applying pressure or external fields, this compound demonstrates non-Fermiliquid behaviour at low temperatures. On lowering the temperature further it also has a superconducting state below $80 \mathrm{mK}$. 
lead to enriched harmonic content in the dHvA oscillations, i.e., the Shoenberg magnetic interaction effect [1]. On looking into this problem, a detailed theory was worked out by Holstein et al. [4]. It provided a firm theoretical basis for this effect, but more importantly predicted new phenomena that were little noticed at the time. Even in the absence of an externally applied magnetic field, it was realized that the long-range current-current forces that gave rise to the Shoenberg effect in finite fields, could destabilize the Fermi-liquid description under certain conditions [4]. This was one of the first predictions of a non-Fermiliquid metal. The direct current-current forces considered by Holstein et al. are however very weak and any nonFermi-liquid behavior would only be expected at temperatures perhaps in the submillikelvin regime. The idea that attractive or long-range effective interactions between quasiparticles can lead to non-Fermi-liquid behavior however continued. Long-range interactions can be routinely achieved by tuning materials to the neighbourhood of a low temperature continuous magnetic quantum phase transition or so-called quantum critical point (QCP). The tuning of materials can be carried out by changing their chemical composition or via the application of pressure or magnetic fields. The interactions between quasiparticles can become non-local via the enhanced magnetic exchange forces near to quantum criticality. Quantum phase transitions exhibit surprisingly subtle and complex behavior, even in comparatively simple examples of cubic ferromagnetic metals of high purity, which will be the main focus of this article. In such cases, one may expect a multiplicity of quantum critical fields and potentially a multiplicity of phase transitions.

\section{The self-consistent field approximation}

Early descriptions of quantum critical points, developed independently for insulating ferroelectric materials [5-7] and ferromagnetic metals [8-12] in the 1970s, were based essentially on $\phi^{4}$ quantum field models. They differ from the Ginzburg-Landau-Wilson models of classical critical phenomena by the inclusion of the dynamics of the order parameter field $\phi(r, \tau)$, which in the case of ferromagnetism represents a coarse-grained magnetic polarization after integrating out Fermionic degrees of freedom. The magnetization field is a function of the spatial coordinate $r$ and temporal coordinate $\tau$ (the imaginary time, which has a finite range at non-zero temperatures, $0<\tau<\hbar / k_{B} T$ ) [13]. The inclusion of the thermal coordinate increases the relevant dimension from the spatial dimension $d$ to the effective dimension $d_{\text {eff }}=d+z$, where $z$ is the dynamical exponent defining the dispersion relation, i.e., the wavevector dependence of the frequency spectrum of fluctuations of the field $\phi$ at small wave vectors. The self-consistent-field approximation, which applies in the case of classical critical phenomena for $d>4$ in the classical $\phi^{4}$ model, might apply under a less restrictive condition $d>4-z$ in the $\phi^{4}$ quantum treatment of critical phenomena. However, as discussed below, there are other more subtle ways in which quantum and classical phase transitions can differ $[14,15]$.

We begin by considering properties of ferromagnetic quantum phase transitions (tuned for example by pressure) that seem capable of a description in the self-consistentfield approximation as outlined above. We then turn to examples of apparent breakdowns of this approximation and discuss the possible reasons for the unexpected behaviors observed. The self-consistent-field approximation to the quantum $\phi^{4}$ field model in a magnetic metal is known as the self-consistent-renormalization (SCR) model, or equivalently the Moriya-Hertz-Millis model [9,12,16,17], which has seen a number of independent and quantitatively different developments [8-12,16-18]. They have in common essentially a mean field decoupling of the interaction between field modes (i.e., the Fourier components $\phi_{q}(\tau)$ of $\phi(r, \tau)$, but differ in some detailed aspects (see, e.g., discussions in Ref. 18). In the calculations presented here we have employed the approach reviewed in Ref. 19.

The spectrum of fluctuations of modes in the metallic state is governed by Landau damping together with the consequences of a vanishing inverse static magnetic susceptibility $\chi^{-1}$, as $T \rightarrow 0$ and the Curie temperature $T_{C} \rightarrow 0$. This leads to a spin-fluctuation spectrum with imaginary frequency, $\Gamma_{q} \sim q^{z}$, where $z=3$. The SCR model has been successful in accounting for a wide range of thermal and transport properties in $d$-electron metals with unsaturated spin polarizations and low $T_{C}$ (see, e.g., $[16,19])$. However, near to and above the critical lattice density where $T_{C} \rightarrow 0$, the SCR model in its simplest form can break down. One of the mechanisms for the breakdown is the emergence of attractive interactions between critical fluctuation modes.

\section{The non-local marginal Fermi liquid state in a weakly ferromagnetic metal}

We now discuss the case of ferromagnetic $d$-electron metals with unsaturated spin polarization and low Curie temperature in more detail. Between $T_{F L}$ and $T_{M F L}$ in the temperature-pressure phase diagram in Fig. 2, the SCR model with $d=3$ reduces approximately to the non-local marginal Fermi liquid (MFL) model [4,12,16,19-21] characterized in particular by a $T^{5 / 3}$ temperature dependence of the electrical resistivity, $\rho$, and $T$-linear temperature dependence of the thermal resistivity, $w$, at low temperature. This is to be contrasted with the predictions of the local marginal Fermi liquid model [22] in which both $\rho$ and $w$ are linear in $T$ at low temperatures. 


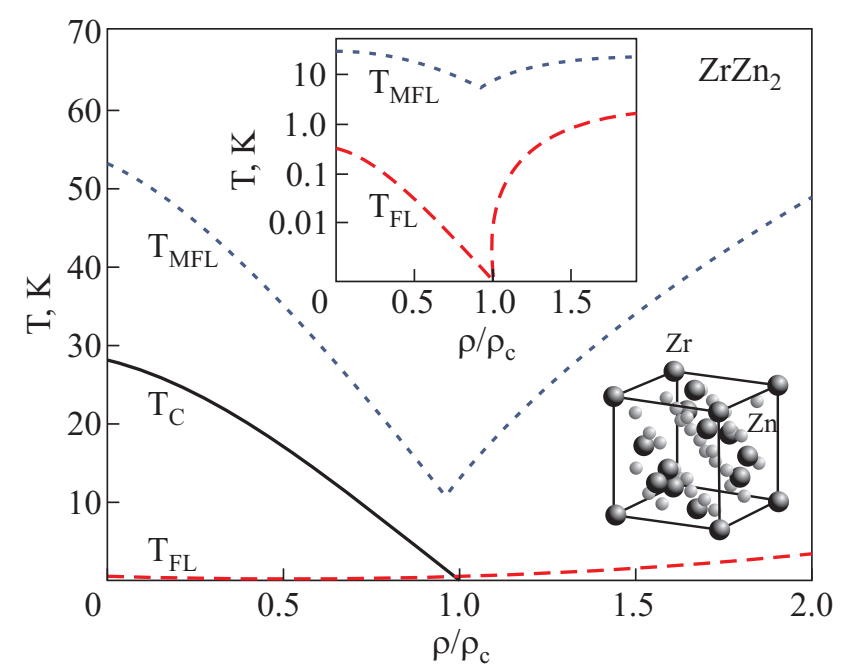

Fig. 2. Temperature-pressure phase diagram predicted by the SCR model for a weakly ferromagnetic metal, $\mathrm{ZrZn}_{2}$ [25]. The solid line, labeled $T_{C}$, is the Curie temperature. Below the lower crossover line, $T_{F L}$, the SCR model predicts Fermi liquid behavior characterized by a $T^{2}$ resistivity. Above $T_{F L}$ up to $T_{M F L}$ (excluding a narrow regime near $T_{C}$ ), the SCR model predicts nonlocal marginal Fermi liquid behavior characterized by a $T^{5 / 3}$ electrical resistivity and $T$-linear thermal resistivity $(d=z=3)$.

The non-local marginal Fermi liquid has been discussed in other contexts [21] and for instance in cases where the relevant critical fields are (i) transverse gauge fields in ideally pure metals as in the theory of Holstein et al. discussed in the introduction [4,20] or (ii) statistical gauge fields on the border of electron localization [23]. In these and other related examples (see e.g. Ref. 24) the dynamical exponent is also three and the temperature dependence of, e.g., the heat capacity is predicted to be of the same form as that of the SCR model for a ferromagnetic quantum critical point.

We consider the example of $\mathrm{ZrZn}_{2}$ that crystallizes in a cubic laves structure (Fig. 2) (see Refs. 25-29 for recent discussions). As shown in Fig. 3, the observed temperature dependences of $\rho$ and $w$ are consistent with the predictions of the SCR model [25]. The correspondence between theory and experiment suggests that the temperature variations of $\rho$ and $w$ at low $T$ are governed mainly by the effects of scattering of carriers from nearly critical ferromagnetic spin fluctuations. Scattering from phonons is found to be sub-dominant below about $15 \mathrm{~K}$ in both $\rho$ and $w$ for samples having residual resistivities well below one $\mu \Omega \mathrm{cm}$ (Ref. 25 supplementary information).

The $T^{5 / 3}$ temperature dependence of $\rho$ is observed to extend up to the critical pressure $p_{c}$ of approximately 20 kbar where $T_{C} \rightarrow 0$ in $\mathrm{ZrZn}_{2}$ (Fig. 4). However, the temperature dependence changes abruptly from $T^{5 / 3}$ to $T^{3 / 2}$ upon crossing $p_{c}$, a result inconsistent with the predictions of the SCR model in its conventional form (inset of Fig. 4) [25,29]. The $T^{3 / 2}$ resistivity extends from $p_{c}$ up to at least twice $p_{c}[29,30]$. Intriguingly, similar behavior, sugges-
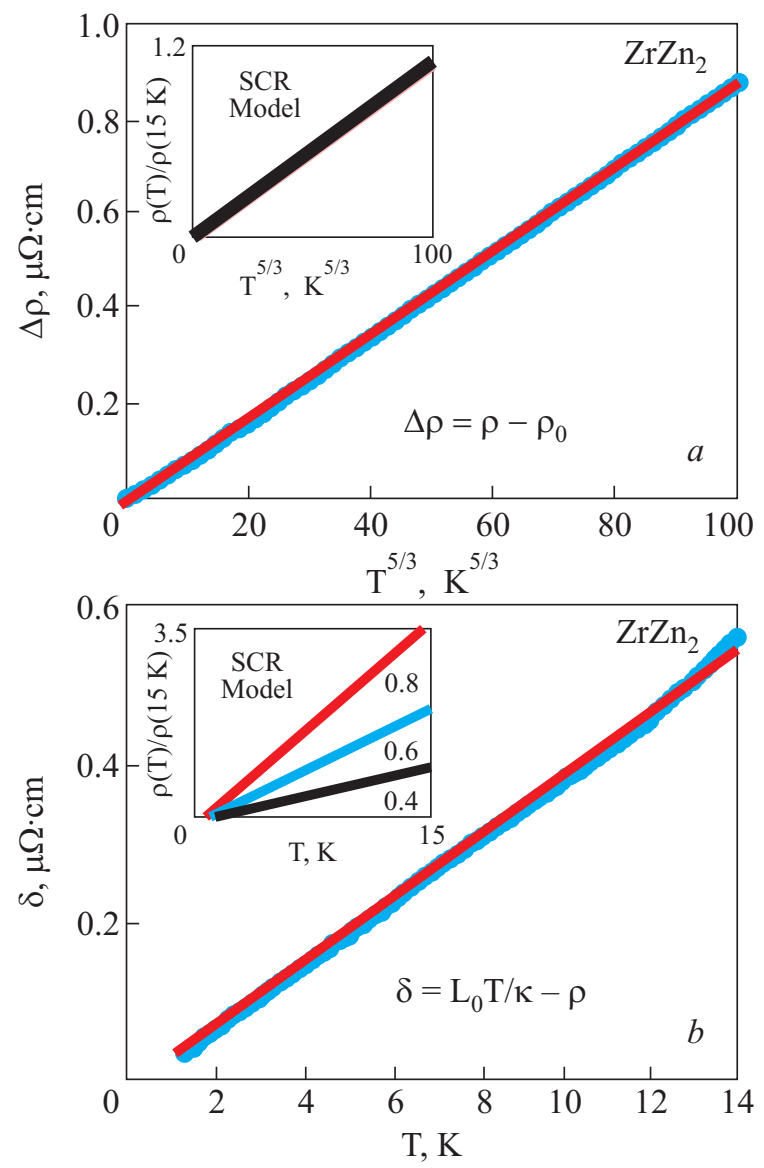

Fig. 3. Temperature dependence of transport properties in a weakly ferromagnetic metal, $\mathrm{ZrZn}_{2}$ [25]: (a) the temperature dependence of the electrical resistivity, $\rho$, and $(b)$ the temperature dependence of the difference $\delta$ between the thermal resistivity $w$ and $\rho$, where $w=L_{0} T / \kappa, \kappa$ is the thermal conductivity and $L_{0}$ is the Lorentz number. The $T^{5 / 3}$ electrical resistivity and $T$-linear thermal resistivities are consistent with the predictions of the SCR model $(d=z=3$, insets in $(a)$ and $(b))$ [16]. The zero temperature model parameters were obtained in a manner similar to that discussed in, e.g., Ref. 19. The cut-off wavevector in the model is taken to be the average radius of the Brillouin zone, and the values given in the inset of $(b)$ correspond to possible characteristic dimensions (in inverse Angstrom) of the relevant Fermi surface sheet. In the pure samples used here with residual resistivities of $0.2 \mu \Omega \mathrm{cm}$ the effect of phonons on $w$ is found to be small and negligible below approximately $15 \mathrm{~K}$.

tive of the existence of a critical regime rather than a critical point, has been reported in other materials on the border of magnetic phase transitions and in particular in $\mathrm{MnSi}$ [31-33] and $\mathrm{YbAlB}_{4}$ [2].

\section{Magnetic inhomogeneities above $p_{c}$}

Both $\mathrm{ZrZn}_{2}$ [34] and MnSi [35] exhibit weak first order transitions just below $p_{c}$ and may be described, along with other materials, by a temperature-pressure-magnetic field phase diagram of the form shown schematically in Fig. 5 $[32,36]$. In this figure a second order transition line falls 


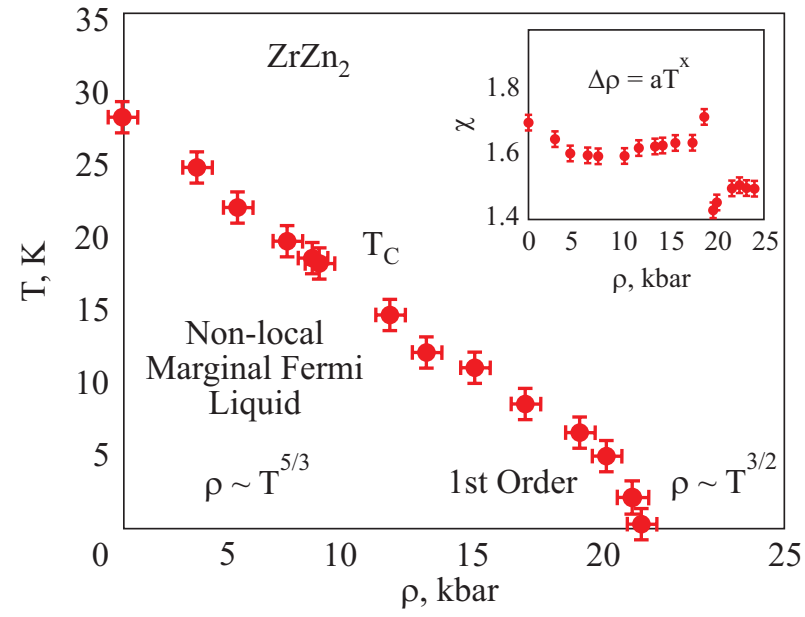

Fig. 4. Temperature-pressure phase diagram of $\mathrm{ZrZn}_{2}$ [25]. The magnetic transition becomes weakly first order near to the critical pressure $p_{c}$ of the order of $20 \mathrm{kbar}$ [34]. The resistivity varies as $T^{5 / 3}$ below $p_{c}$ and as $T^{3 / 2}$ above $p_{c}$ (upper inset) (see also Refs. 27, 29). The $T^{3 / 2}$ resistivity, found to extend from $p_{c}$ to at least twice $p_{c}$ within the temperature range $1 \mathrm{~K}$ to $15 \mathrm{~K}[29,30]$, is inconsistent with the predictions of the SCR model in its conventional form.

with increasing pressure and bifurcates at a tricritical point leading to two sheets of first order transitions (the negative magnetic field sheet is not shown).

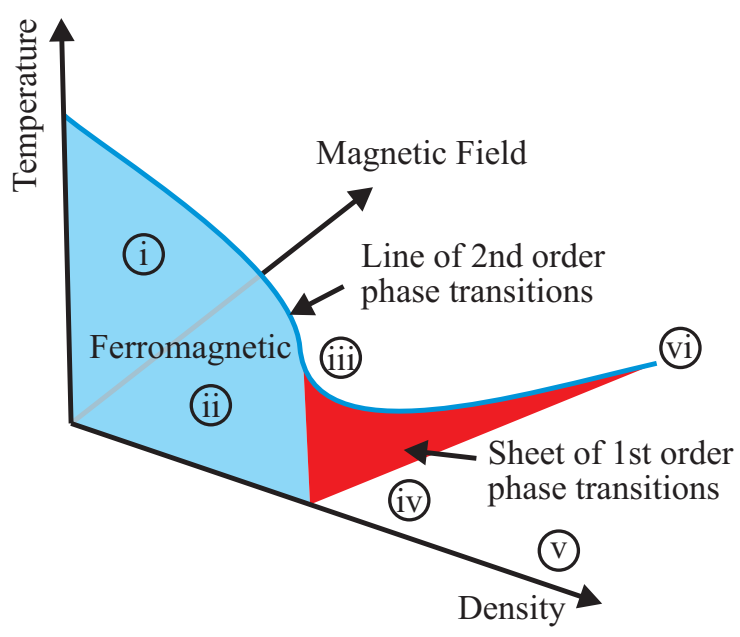

Fig. 5. Temperature-pressure-magnetic field phase diagram on the border of ferromagnetism. Qualitative form of the phase diagram predicted by the $\phi^{6} \mathrm{SCR}$ model with an attractive $\phi^{4}$ term in the effective action. Selected examples of phenomena observed on the border of ferromagnetism are marked on the phase diagram - (i) the marginal Fermi liquid state in $\mathrm{ZrZn}_{2}$ [25], (ii) an example of the coexistence of superconductivity and metallic ferromagnetism ( $\mathrm{UGe}_{2}$ [53-55]), (iii) quantum tri-criticality in $\mathrm{Ni}_{3} \mathrm{Ga}$ [56], (iv) spin textured phase in $\mathrm{MnSi}[31,32,36]$, (v) spin-triplet superconductivity on the border of ferromagnetism $\left(\mathrm{M}_{2} \mathrm{RuO}_{4}\right.$, where $\mathrm{M}$ stands for $\mathrm{Sr}[57,58]$ or potentially $\mathrm{Ca}$ at high pressures [59]) and (vi) an electronic nematic phase in $\mathrm{Sr}_{3} \mathrm{Ru}_{2} \mathrm{O}_{7}[60,61]$ near to a quantum critical end point in high magnetic fields.

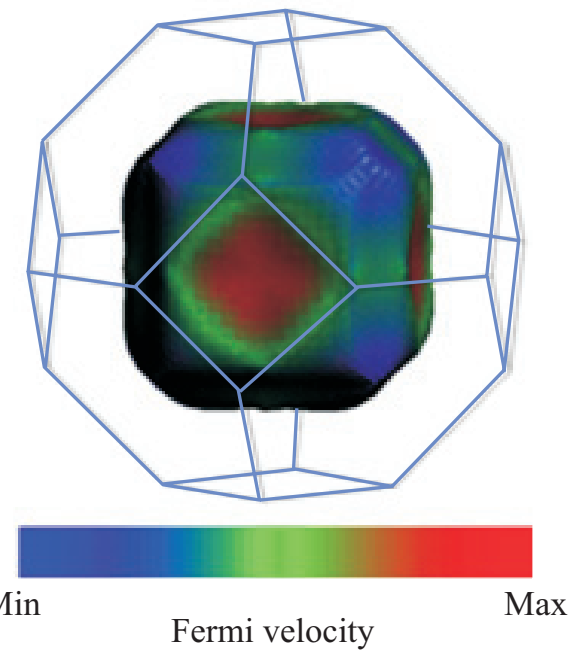

Fig. 6. Calculated Fermi surface sheet of $\mathrm{TiBe}_{2}$ [50], which is expected to be similar to the Fermi surface of $\mathrm{ZrZn}_{2}$ [26] in the paramagnetic state (the electron-"pseudocube" is shown only). Calculations of the non-interacting susceptibility (Lindhart function) suggest that the magnetic properties of $\mathrm{ZrZn}_{2}$ arise from the proximity of the corners of the electron-"pseudocube" and also across the hole-"jungle-gym" (not shown) [50]. These features are expected to lead to enhanced ferromagnetic and antiferromagnetic spin fluctuations in the region near to the tricritical point in Fig. 5.

The state near to the tricritical point in materials such as $\mathrm{MnSi}$ is characterized by slowly varying magnetic inhomogeneities not described by the SCR model in its conventional form [37-39]. The existence of a tricritical point and magnetic inhomogeneities near to and above $p_{c}$ has been attributed, for example, to (i) the magneto-elastic coupling [40], (ii) anharmonic quantum precession of the magnetization $[16,19,41]$ and (iii) non-analytic corrections to the magnetic equation of state expected to arise when full account is taken of the effects of gapless particle-hole excitations at the Fermi surface [42-47]. These effects lead to attractive interactions between spin fluctuation modes and to a phase diagram of the form shown in Fig. 5. Potentially they also lead to intrinsic magnetic inhomogeneities near to $p_{c}$.

In principle, first order transitions and inhomogeneities can also arise via the effects of van Hove and nesting singularities of the Fermi surface [16,48-51]. A Fermi surface such as that predicted for paramagnetic $\mathrm{ZrZn}_{2}$, that is characterized both by a low Fermi velocity at $k$-points near to van Hove singularities along $<111>$ directions and strong nesting along $<100>$ directions, would be consistent with the existence of enhanced ferromagnetic as well as antiferromagnetic spin fluctuations [50,51]. See for example the calculated Fermi surface of $\mathrm{TiBe}_{2}$ in Fig. 6 [50] which is expected to be similar to the Fermi surface of $\mathrm{ZrZn}_{2}$ in the paramagnetic phase [26,51]. These features could lead to the existence of two or more quasi-critical fields and to a 
state that is more inhomogeneous than expected in the presence of ferromagnetic fluctuations alone. We also note that such a Fermi surface model can lead to a first order transition to ferromagnetism at sufficiently small lattice density and to a phase diagram of the form shown in Fig. 5 [48-51]. A more recent example in which these effects may be important is reported in Ref. 52.

\section{Additional phase transitions}

A study of the simplest magnetic $d$-electron metals reveals that the border of ferromagnetism appears to be characterized by a multiplicity of quasi-critical fields and potentially a multiplicity of phase transitions (Fig. 5). The border of ferromagnetism can thus be more intriguing than was generally envisaged in the early work on quantum critical phenomena. The occurrence of a multiplicity of quantum critical fields is not limited to the problems that we have considered but appears to be a recurrent theme in the study of quantum phase transitions in general.

For instance, the case of high $T_{c}$ cuprates is thought to involve a plethora of neighbouring phases, including antiferromagnetism, electron nematic order, $d$-wave superconductivity and perhaps multiple quantum liquid states on the border of Mott transitions. The $f$-electron heavy-fermion systems can also exhibit analogous remarkable phenomena on the edge of $f$-electron localization. The diverse views reported on the nature of $f$-electron heavy fermion systems related in part to the magnetic metals discussed here can be inferred for example from Refs. 23, 24, 62-73. The $f$ electron systems display not only a multiplicity of quantum critical fields, but also show evidence of scaling behaviours not expected in terms of the early models of quantum critical phenomena (see, e.g., Refs. 74-76).

Another area of interest is the case of quantum criticality in displacive ferroelectrics such as $\mathrm{SrTiO}_{3}$ [77,78]. In these systems the self-consistent field model predicts correctly in first approximation the observed $1 / T^{2}$ form of the dielectric constant and thus the breakdown of the traditional Curie law on the border of the quantum critical point. However, broad low temperature peaks in the dielectric constant on the paraelectric side of the phase diagram [77] and evidence in ferroelectric $\mathrm{SrTi}^{18} \mathrm{O}_{3}$ [79], indicate that the effective bosonic fields may become attractive in the neighborhood of the QCP leading to a potential phase diagram similar to that shown in Fig. 5. This is therefore rather similar behavior to that seen in the ferromagnetic metals discussed above.

During the early developments of the understanding of metallic magnetism over a century ago, it was realized that the direct spin-spin interactions were too weak to account for the observed Curie temperatures. Instead it was found that magnetism comes about via effective exchange interactions with their origins in the Coulomb repulsion and Pauli exclusion principles (equivalently thought of as an

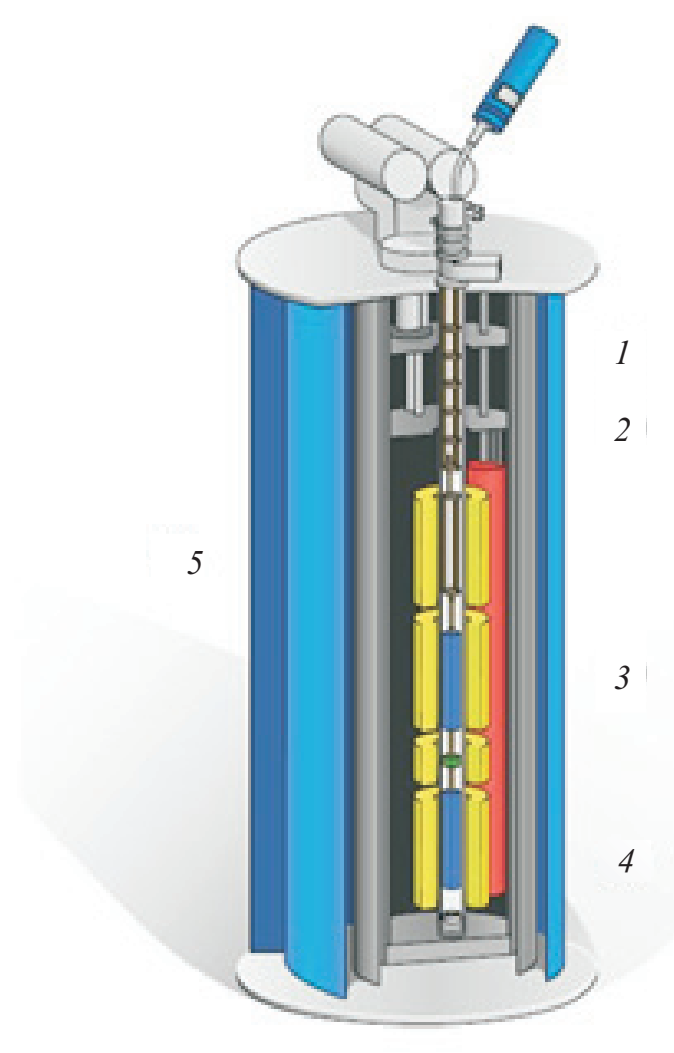

Fig. 7. Surveying quantum phase transitions. Cryogen-free automated system for scanning over wide ranges in temperature, magnetic field and pressure. The figure illustrates the cryomagnetic system being developed in a collaboration between Dryogenics Ltd and the Cavendish Laboratory for routine scans from room temperature to the low millikelvin range employing a pulse tube cryocooler (40 K and $4 \mathrm{~K}$ plates on top (1) and (2)) and a twostage magnetic refrigerator (two magnets and two demagnetization pills at bottom (3) and (4)). The experimental region (with surrounding magnet in middle (5)) accommodates a variablepressure diamond-anvil cell designed for automated electronic and magnetic measurements under quasi-hydrostatic conditions.

effective attraction between particles and holes in a zero angular momentum state). In fact, the original currentcurrent interactions considered by Holstein et al. [4] and at the beginning of this article, may also be greatly enhanced in an analogous way. This brings about the possibility of observing ordered or fluctuating charge current states in metallic systems. Such ideas have been considered in many works (e.g. Ref. 80) and provide a way of thinking about pairing of particles and holes in many-body states with anisotropic energy gaps analogous to anisotropic gaps in unconventional superconductors.

Detailed examinations of quantum phase transitions require scans of broad regions of phase space by the precise and painstaking control of tuning parameters including temperature, pressure, magnetic field, electric field, and materials properties [81]. An automated cryogen-free measurement system that may help to facilitate studies of 
quantum phase transitions and the search for novel phenomena in the future is illustrated in our concluding figure (Fig. 7). This system, which is currently under development, allows the temperature to be changed between room temperature and the low millikelvin range with built-in pressure and magnetic field control technology. This allows the option of varying the pressure in situ with a diamond anvil cell at low temperatures for fully automated scans over wide ranges of temperature, magnetic field and pressure.

We are grateful for discussions with P. Chandra, P. Coleman, G.J. Conduit, F.M. Grosche, R.K.W. Haselwimmer, D.E. Khmelnitskii, P. Littlewood, A.P. Mackenzie, P. Monthoux, J.F. Scott, L. Sibley, B.D. Simons, D. Tompsett, I.R. Walker, and J. Wensley. We also thank S.V. Brown, G.L. Lonzarich, and D. Astill for their invaluable assistance. The Engineering and Physical Sciences Research Council of the United Kingdom, the Royal Society of London, the Isaac Newton Trust, and Jesus, Emmanuel and Trinity Colleges of the University of Cambridge have supported this work.

1. D. Shoenberg, Magnetic oscillations in metals, Cambridge University Press, Cambridge (1984).

2. S. Nakatsuji, K. Kuga, Y. Machida, T. Tayama, T. Sakakibara, Y. Karaki, H. Ishimoto, S. Yonezawa, Y. Maeno, E. Pearson, G.G. Lonzarich, L. Balicas, H. Lee, and Z. Fisk, Nat. Phys. 4, 603 (2008).

3. E.C. O'Farrell, D.A. Tompsett, S.E. Sebastian, N. Harrison, C. Capan, L. Balicas, K. Kuga, A. Matsuo, K. Kindo, M. Tokunaga, S. Nakatsuji, G. Csányi, Z. Fisk, and M.L. Sutherland, Phys. Rev. Lett. 102, 216402 (2009).

4. T. Holstein, R.E. Norton, and P. Pincus, Phys. Rev. B8, 2649 (1973).

5. A.B. Rechester, Sov. Phys. JETP 33, 423 (1971).

6. D.E. Khmelnitskii and V.L. Shneerson, Sov. Phys. Solid State 13, 687 (1971).

7. D.E. Khmelnitskii and V.L. Shneerson, Sov. Phys. JETP 37, 164 (1973).

8. K.K. Murata and S. Doniach, Phys. Rev. Lett. 29, 285 (1972).

9. T. Moriya, and A. Kawabata, J. Phys. Soc. Jpn. 34, 639 (1973).

10. T.V. Ramakrishnan, Phys. Rev. B10, 4014 (1974).

11. I.E. Dzyaloshinski, Sov. Phys. JETP 43, 1036 (1976).

12. J.A. Hertz, Phys. Rev. B14, 1165 (1976).

13. S. Sachdev, Quantum Phase Transitions, Cambridge University Press, Cambridge (1999).

14. P.W. Anderson, Physica B318, 28 (2002).

15. R.B. Laughlin, G.G. Lonzarich, P. Monthoux, and D. Pines, Adv. Phys. 50, 361 (2001).

16. T. Moriya, Spin Fluctuations in Itinerant Electron Magnetism, Springer, Berlin (1985).

17. A.J. Millis, Phys. Rev. B48, 7183 (1993).

18. G.G. Lonzarich and L. Taillefer, J. Phys. C18, 4339 (1985).
19. Electron: a Centenary Volume, G.G. Lonzarich and M. Springford (eds.), Cambridge University Press, Cambridge (1997), Ch. 6.

20. M.Y. Reizer, Phys. Rev. B39, 1602 (1989).

21. G. Baym and C. Pethick, Landau Fermi-Liquid Theory: Concepts and Applications, Wiley, N.Y. (1991).

22. C.M. Varma, P.B. Littlewood, S. Schmitt-Rink, E. Abrahams, and A.E. Ruckenstein, Phys. Rev. Lett. 63, 1996 (1989).

23. T. Senthil, M. Vojta, and S. Sachdev, Phys. Rev. B69, 035111 (2004).

24. I. Paul, C. Pepin, and M.R. Norman, Phys. Rev. Lett. 98, 026402 (2007).

25. R.P. Smith, M. Sutherland, G.G. Lonzarich, S.S. Saxena, N. Kimura, S. Takashima, M. Nohara, and H. Takagi, Nature 455, 1220 (2008).

26. S.J.C. Yates, G. Santi, S.M. Hayden, P.J. Meeson, and S.B. Dugdale, Phys. Rev. Lett. 90, 057003 (2003).

27. E.A. Yelland, S.M. Hayden, S.J.C. Yates, C. Pfleiderer, M. Uhlarz, R. Vollmer, H. von Löhneysen, N.R. Bernhoeft, R.P. Smith, S.S. Saxena, and N. Kimura, Phys. Rev. B72, 214523 (2005).

28. D.A. Sokolov, M.C. Aronson, W. Gannon, and Z. Fisk, Phys. Rev. Lett. 96, 116404 (2006).

29. S. Takashima, M. Nohara, H. Ueda, N. Takeshita, C. Terakura, F. Sakai, and H. Takagi, J. Phys. Soc. Jpn. 76, 043704 (2007).

30. L. Sibley, and J. Wensley, Private Communications (2009).

31. C. Pfleiderer, S.R. Julian, and G.G. Lonzarich, Nature 414, 427 (2001).

32. N. Doiron-Leyraud, I.R. Walker, L. Taillefer, M.J. Steiner, S.R. Julian, and G.G. Lonzarich, Nature 425, 595 (2003).

33. P. Pedrazzini, D. Jaccard, G. Lapertot, J. Flouquet, Y. Inada, H. Kohara, and Y. Onuki, Physica B378-80, 165 (2006).

34. M. Uhlarz, C. Pfleiderer, and S.M. Hayden, Phys. Rev. Lett. 93 (2004).

35. C. Pfleiderer, G.J. McMullan, S.R. Julian, and G.G. Lonzarich, Phys. Rev. B55, 8330 (1997).

36. D. Belitz, T.R. Kirkpatrick, and J. Rollbuhler, Phys. Rev. Lett. 94 (2005).

37. W. Yu, F. Zamborszky, J.D. Thompson, J.L. Sarrao, M.E. Torelli, Z. Fisk, and S.E. Brown, Phys. Rev. Lett. 92, 086403 (2004).

38. C. Pfleiderer, D. Reznik, L. Pintschovius, H. von Lohneysen, M. Garst, and A. Rosch, Nature 427, 227 (2004).

39. Y.J. Uemura, A.A. Azcel, Y Ajiro, J.P. Carlo, T. Goko, D.A. Goldfield, A Kitada, and G.M. Luke, Nat. Phys. 3, 29 (2007).

40. G.A. Gehring, Europhys. Lett. 82, 60004 (2008).

41. M.T. Beal-Monod, Phys. Rev. Lett. 20, 929 (1968).

42. S. Misawa, Phys. Rev. Lett. 26, 1632 (1971).

43. G. Barnea and D.M. Edwards, J. Phys. F7, 1323 (1977).

44. D. Belitz, T.R. Kirkpatrick, and T. Vojta, Phys. Rev. B55, 9452 (1997).

45. G.Y. Chitov and A.J. Millis, Phys. Rev. Lett. 86, 5337 (2001). 
46. A.V. Chubukov and D.L. Maslov, Phys. Rev. B69, 121102(R) (2004)

47. G.J. Conduit, A.G. Green, and B.D. Simons, Phys. Rev. Lett. 103, 207201 (2009).

48. J. Kubler, Phys. Rev. B70, 024407 (2004).

49. Mazin, II, and D.J. Singh, Phys. Rev. B69, 064429 (2004).

50. T. Jeong, A. Kyker, and W.E. Pickett, Phys. Rev. B73, 115106 (2006).

51. D. Tompsett, Private Communications (2009).

52. M. Brando, W.J. Duncan, D. Moroni-Klementowicz, C. Albrecht, D. Grüner, R. Ballou, and F.M. Grosche, Phys. Rev. Lett. 101, 026401 (2008).

53. S.S. Saxena and P. Agrapwal, Nature 406, 587 (2000).

54. A. Harada, S. Kawasaki, H. Mukuda, Y. Kitaoka, Y. Haga, E. Yamamoto, Y. Ōnuki, K.M. Itoh, E.E. Haller, and H. Harima, Phys. Rev. B75, 140502 (2007).

55. A.D. Huxley, S.J.C. Yates, F. Lévy, and I. Sheikin, J. Phys. Soc. Jpn. 76, 051011 (2007).

56. R.P. Smith, J. Phys. Condens. Matter 21, 095601 (2009).

57. Y. Maeno, H. Hashimoto, K. Yoshida, S. Nishizaki, T. Fujita, J.G. Bednorz, and F. Lichtenberg, Nature 372, 532 (1994).

58. A.P. Mackenzie and Y. Maeno, Rev. Mod. Phys. 75, 657 (2003).

59. P.L. Alireza, J. Gillett, Y.T. Chris Ko, S.E. Sebastian, and G.G. Lorezarich, J. Phys. Condens. Matter 22, 052201 (2010).

60. S.A. Grigera, R.S. Perry, A.J. Schofield, M. Chiao, S.R. Julian, G.G. Lonzarich, S.I. Ikeda, Y. Maeno, A.J. Millis, and A.P. Mackenzie, Science 294, 329 (2001).

61. R.A. Borzi, S.A. Grigera, J. Farrell, R.S. Perry, S.J.S. Lister, S.L. Lee, D.A. Tennant, Y. Maeno, and A.P. Mackenzie, Science 315, 214 (2007).

62. Y. Ōnuki, R. Settai, K. Sugiyama, T. Takeuchi, T.C. Kobayashi, Y. Haga, and E. Yamamoto, J. Phys. Soc. Jpn. 73, 769 (2004).

63. P. Coleman and A.J. Schofield, Nature 433, 226 (2005).

64. A. Schofield, Phys. World 16, 23 (2003).
65. M.B. Maple, N.A. Frederick, P.C. Ho, W.M. Yuhasz, and T. Yanagisawa, J. Supercond. Nov. Magn. 19, 299 (2006).

66. G.R. Stewart, Rev. Mod. Phys. 73, 797 (2001).

67. G.R. Stewart, Rev. Mod. Phys. 78, 743 (2006).

68. P. Gegenwart, T. Westerkamp, C. Krellner, Y. Tokiwa, S. Paschen, C. Geibel, F. Steglich, E. Abrahams, and Q. Si, Science 315, 969 (2007).

69. H. von Löhneysen, A. Rosch, M. Vojta, and P. Wölfle, Rev. Mod. Phys. 79, 1015 (2007).

70. P. Monthoux, D. Pines, and G.G. Lonzarich, Nature 450, 1177 (2007).

71. Y.F. Yang, Z. Fisk, H.O. Lee, J. D. Thompson, and D. Pines, Nature 454, 611 (2008)

72. S. Friedemann, T. Westerkamp, M. Brando, N. Oeschler, S. Wirth, P. Gegenwart, C. Krellner, C. Geibel, and F. Steglich, Nat. Phys. 5, 465 (2009).

73. J. Flouqet and H. Harima, arXiv:0910.3110v2 (2009).

74. M.C. Aronson, R. Osborn, R.A. Robinson, J.W. Lynn, R. Chau, C.L. Seaman, and M.B. Maple, Phys. Rev. Lett. 75, 725 (1995).

75. A. Schröder, G. Aeppli, R. Coldea, M. Adams, O. Stockert, H. von Löhneysen, E. Bucher, R. Ramazashvili, and P. Coleman, Nature 407, 351 (2000).

76. Q.M. Si, K. Ingersent, and J.L. Smith, Nature 413, 804 (2001).

77. S.E. Rowley, L.J. Spalek, R.P. Smith, M.P.M. Dean, G.G. Lonzarich, J.F. Scott, and S.S. Saxena, arXiv:0903.1445 (2009).

78. P. Coleman, Physica B378-80, 1160 (2006).

79. T. Shigenari, K. Abe, T. Takemoto, O. Sanaka, T. Akaike, Y. Sakai, R. Wang and M. Itoh, Phys. Rev. B74, 174121 (2006).

80. C. Honerkamp, M. Salmhofer, and T.M. Rice, Eur. Phys. J. B27, 127 (2002).

81. P.L. Alireza, Y.T.C. Ko, J. Gillett, C.M. Petrone, J.M. Cole, S.E. Sebastian, and G.G. Lonzarich, J. Phys. Condens. Mat2ter 21, 012208 (2009). 